\title{
Current driven vortex-antivortex pair breaking and vortex explosion in the $\mathrm{Bi}_{2} \mathrm{Te}_{3} / \mathrm{FeTe}$ interfacial superconductor
}

\author{
C. L. Dean ${ }^{\mathrm{a}}$, M. N. Kunchur ${ }^{\mathrm{a}}$, Q. L. He ${ }^{\mathrm{b}}$, H. Liu ${ }^{\mathrm{b}}$, J. Wang ${ }^{\mathrm{b}}$, R. Lortz ${ }^{\mathrm{b}}$, I. K. \\ $\mathrm{Sou}^{\mathrm{b}}$ \\ ${ }^{a}$ Department of Physics and Astronomy, University of South Carolina, Columbia, SC \\ 29208, U.S.A. \\ ${ }^{b}$ William Mong Institute of Nano Science and Technology, the Hong Kong University of \\ Science and Technology, Hong Kong, China
}

\begin{abstract}
We investigated the dissipative regime of the $\mathrm{Bi}_{2} \mathrm{Te}_{3} / \mathrm{FeTe}$ topological insulatorchalcogenide interface superconductor at temperatures well below the BerezinskiKosterlitz-Thouless transition. We observe a transition in the current-resistance and temperature-resistance curves that quantitatively agrees with the Likharev vortex-explosion phenomenon. In the limit of low temperatures and high current densities, we were able to demonstrate the regime of complete vortex-antivortex dissociation arising from current driven vortex-antivortex pair breaking.
\end{abstract}

\section{Introduction}

A principal cause of dissipation and resistance within the superconducting state is the motion of flux vortices. In thin film samples, vortices parallel to the film plane do not contribute significantly to the dissipation because of very strong intrinsic pinning. Thus dissipation arises principally from vortices perpendicular to the film plane. In the absence of an applied magnetic field, in thin samples, vortices perpendicular to the sample plane can be generated spontaneously by thermal fluctuations through two main routes: (1) the unbinding of virtual vortex-antivortex pairs generated in the interior of the sample and (2) unbinding of single vortices spontaneously generated at the sample edge from their image antivortices just outside the edge.

The first scenario, which requires the film to be sufficiently thin as to be in the two-dimensional limit $(\xi<d$, where $\xi$ is the superconducting coherence length and $d$ is the film thickness), corresponds to the well known BKT (Berezinski-Kosterlitz-Thouless) state $[1,2,3,4,5,6,7,8]$ that appears above a temperature $T_{B K T}$ where one has a plasma of thermally unbound vortices

Email address: kunchur@sc.edu (M. N. Kunchur)

URL: http://www.physics.sc.edu/ ${ }^{\sim}$ kunchur (M. N. Kunchur)

Preprint submitted to Elsevier

February 27, 2016 
of both polarities. Besides temperature, vortex-antivortex pairs can also be unbound by the Lorentz force of an applied transport current.

The second scenario, the edge nucleation of single vortices as proposed recently by Gurevich and Vinokur (GV) [9], can become a dominant source of dissipation under certain conditions (e.g., somewhat outside the $2 \mathrm{D}$ limit $\xi>d$ and for $T<T_{B K T}$ ) for which the BKT dissipation is suppressed. As shown previously [10], the GV edge nucleation process is in turn facilitated when parallel vortex segments are precluded by the Likharev vortex explosion phenomenon [11]. The gist of the Likharev phenomenon is that a stable vortex core can exist inside a film along the direction parallel to the film only if there is a minimum thickness $d \geq 4.4 \xi(T)$. As one or both dimensions transverse to the vortex core are progressively reduced, the circulating supercurrents around the core become squeezed leading to an increase in the local current density. This increases current induced pair breaking, which suppresses the order parameter leading to an increase in coherence length. This causes the vortex to expand and further squeeze the circulating supercurrents. This self feeding process causes the vortex core to explode below some critical dimension $4.4 \xi(T)$. Conversely if the film thickness is fixed (which is usually the case) with increasing temperature the vortex will explode at a certain vortex-explosion temperature $T_{v}$ which satisfies $\xi\left(T_{v}\right)>d / 4.4$. Our previous work on thin molybdenum-germanium (MoGe) films [10] provided the first experimental confirmation of this prediction.

In this work we studied the current-resistance and temperature-resistance curves in the $\mathrm{Bi}_{2} \mathrm{Te}_{3} / \mathrm{FeTe}$ interface superconductor. In the limit of low temperatures $\left(T \ll T_{B K T}\right)$ and high pulsed current densities $j$ (but with $j \ll j_{d}$ the depairing current density), we are able to reach the condition that corresponds to the unbinding of the entire population of vortex-antivortex pairs (similar to when $\left.T>T_{B K T}\right)$. We also observed at low currents, the drop in dissipation related to the Likharev vortex explosion. As found in our earlier work, the explosion is sensitive to the conditions of the measurement, and gets washed out by noise, magnetic field, and higher levels of measuring current. To our knowledge this is one of only two experiments that have shown evidence of the Likharev vortex explosion, a phenomenon that was theoretically predicted more than thirty years ago. The measurement also sheds light on the thickness of the superconducting layer. The interface of the $\mathrm{Bi}_{2} \mathrm{Te}_{3} / \mathrm{FeTe}$ heterostructure represents the first realization of superconductivity at the interface between a topological insulator $\left(\mathrm{Bi}_{2} \mathrm{Te}_{3}\right)$ and an iron-chalcogenide (FeTe). Many questions remain as to the origin and nature of superconductivity at the interface, since neither system is a superconductor by itself [12]. One of the crucial questions is regarding the thickness of the interface that carries the superconductivity. In their work, He et al. observed certain signatures of the BKT transition and a square-root temperature dependence of the parallel upper-critical-field, which allowed them to conclude that the $2 \mathrm{D}$ superconductivity resides in a $d=7$ $\mathrm{nm}$ thick interface layer. The present work provides independent information indicating that the superconducting layer is indeed 7-8 $\mathrm{nm}$ thick. 


\section{Experimental details}

The sample was synthesized by a VG-V80H MBE system, and consist of a ZnSe buffer layer $(50 \mathrm{~nm})$ deposited on a GaAs (001) semi-insulating substrate, followed by a deposition of $220 \mathrm{~nm}$ thick FeTe, which is then capped with a 20 $\mathrm{nm}$ thick $\mathrm{Bi}_{2} \mathrm{Te}_{3}$ layer (This thickness comprises 20 QLs, i.e., quintuple layers. The $\mathrm{Bi}_{2} \mathrm{Te}_{3}$ unit cell consists of 3 QLs bonded by van der Waals forces along the [0001] direction.) Upper-critical-field measurements [12] indicate that the superconductivity occurs within a $7 \mathrm{~nm}$ thick interfacial layer, which is much thinner than both the FeTe and $\mathrm{Bi}_{2} \mathrm{Te}_{3}$ layers.

The present work utilized projection photolithography followed by argonion milling to pattern a microbridge with lateral dimensions of width $w=$ $11.5 \mu \mathrm{m}$ and length $l=285 \mu \mathrm{m}$. The onset $T_{c}$ (defined as the intersection of the extrapolation of the normal-state portion and the extrapolation of the steep transition portion of the $R(T)$ curve) was $11.7 \mathrm{~K}$. Further details about sample preparation are provided elsewhere [12].

The cryostat was a Cryomech PT405 pulsed-tube closed-cycle refrigerator that went down to about $3.7 \mathrm{~K}$. All measurements are conducted in zero magnetic field. The majority of the electrical transport measurements used continuous dc currents in the standard four-probe current-direction-reversed configuration with the voltage measured by a Keithley model $2182 \mathrm{~A}$ nanovoltmeter. The variable current curves used a programmable Hewlett Packard 5532A dc power supply. The single fixed low-current temperature-resistance curve used a battery-based current source to reduce noise to a minimum. The dc technique is generally better suited for small-signal measurements, where it provides a better signal-to-noise ratio. The large-signal data were measured with a pulsed technique to reduce sample heating. These pulsed measurements used in-house built pulsed current sources, preamplifier circuitry, and a LeCroy model 9314A digital storage oscilloscope. The pulse durations were in the $0.1-5 \mu$ s range with a pulse repetition frequency of $\sim 1 \mathrm{~Hz}$ (duty cycles of $\sim 1$ part per $10^{6}$ ). This reduces Joule heating of the sample to the $\mathrm{mK}$ range, as was ascertained by a direct measurement of the thermal resistance $\Delta T / \Delta P \approx 0.4 \mathrm{~K} / \mathrm{W}$ using the method devised in our previous work [13]. About 100 pulses were averaged to improve the signal-to-noise ratio. Further details of the pulsed measurement techniques have been published in previous review articles $[14,15,16]$.

\section{Results and discussion}

Fig. 1(a) shows the extended $R(I)$ (resistance-vs-current) response, with the lower portion measured using continuous dc currents and the higher portion measured using fast pulsed signals. The temperature for these data is $T=10.5$ $\mathrm{K}$, which is above $T_{B K T}$. On the left of the graph, notice that the resistance plateaus to a finite value as $I \rightarrow 0$, indicative of an Ohmic response. This is expected because of the plasma of unbound vortices and antivortices that exists in thermal equilibrium above $T_{B K T}$, even in the absence of the driving force of a current. Fig. 1 (b) shows similar curves at $T=3.7 \mathrm{~K} \ll T_{B K T}$. In this 

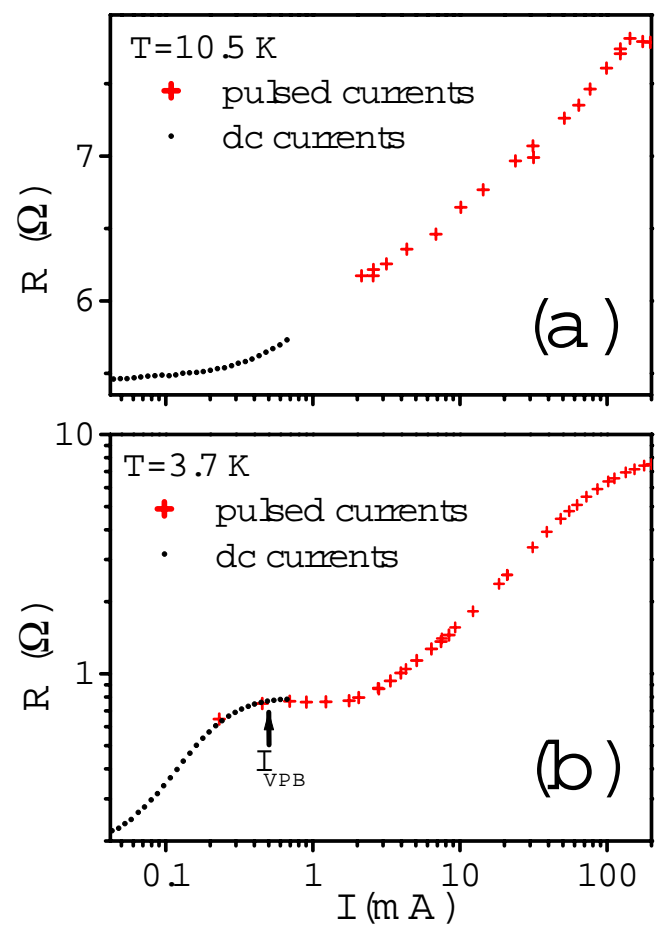

Figure 1: (a) $R \neq 0$ as $I \rightarrow 0$ for $T>T_{B K T}$. (b) $R \rightarrow 0$ as $I \rightarrow 0$ for $T<T_{B K T}$; however, increasing $I$ above the threshold of $I_{V P B} \approx 0.5 \mathrm{~mA}$ (arrow) restores an Ohmic $R \neq 0$ plateau due to the breaking of vortex-antivortex pairs by the Lorentz force of the current. We define this $I_{V P B}$ as the "vortex-pair-breaking current".

case $R \rightarrow 0$ as $I \rightarrow 0$, instead of reaching an Ohmic plateau, indicating that the vortex-antivortex pairs become bound and non-dissipative as the separating force of the current vanishes, since purely thermal dissociation vanishes for $T<T_{B K T}$. Tracing this $R(I)$ curve from lowest to highest $I$, the resistance rises with increasing $I$ and momentarily saturates to a constant value in the middle of the graph above $I \approx 0.5 \mathrm{~mA}$. In anticipation that this plateau corresponds to the condition when most of the vortex-antivortex pair population has become unbound from the force of the current, we will call this quantity $I_{V P B} \approx 0.5$ $\mathrm{mA}$, the "vortex-pair-breaking current". We obtain an estimate of $I_{V P B}$ by starting with the inter-vortex potential $[17,18]$

$$
U=\frac{d}{2 \pi \mu_{0}}\left(\frac{\Phi_{0}}{4 \pi \lambda}\right)^{2}\left(\ln \frac{\left|\vec{r}-\overrightarrow{r^{\prime}}\right|}{\xi}-\ln \frac{w}{\xi}\right),
$$

where $\lambda$ is the magnetic penetration depth and $\Phi_{0}=h / 2 e$ is the flux quantum. The current density exerts a constant force $j d \Phi_{0}$ on the vortex, adding a poten- 
tial $-j d \Phi_{0} r$ to Eq.(1). Defining the dimensionless interaction $u=\frac{U d}{\pi \mu_{0}}\left(\frac{\Phi_{0}}{4 \pi \lambda}\right)^{2}$, and the dimensionless length $x=\left|\vec{r}-\overrightarrow{r^{\prime}}\right| / \xi$, and including a factor $x /(x+\xi)$ as a cutoff of the vortex-antivortex interaction at distances of the order of the coherence length, we can express the interaction as

$$
u=\frac{1}{2}\left(\frac{x}{x+1}\right)\left(\ln \frac{x}{\ell}\right)-k x,
$$

where $\ell=w / \xi$ and $k=j \xi \pi \mu_{0}(4 \pi \lambda)^{2} / \Phi_{0}$. For $k=0$, this has a single minimum at $x=0.718$ (i.e., for $\left|\vec{r}-\overrightarrow{r^{\prime}}\right|<\xi$ ), which represents the equilibrium separation of the paired vortices at zero current. For $k>0$, the potential has a maximum for $x>1$ outside the core and goes to $-\infty$ beyond this peak. The vortices could dissociate by being thermally activated over or by tunneling through this peak. As the current $(k)$ is increased, the peak becomes progressively reduced until it finally merges with the minimum. At this point the vortices are no longer bound, and this current can be interpreted as $I_{V P B}$. A numerical calculation shows that this happens at $k=0.128$. Taking $\lambda=124 \times 10^{-9} \mathrm{~m}$ and $\xi=$ $4.4 \times 10^{-9} / \mathrm{m}[12,19]$, this translates to a current density $j=6.28 \times 10^{9}$ $\mathrm{A} / \mathrm{m}^{2}$. For our sample dimensions of $w=11.6 \mu \mathrm{m}$ and $d=7 \mathrm{~nm}$, we get a current $I_{V P B}=0.509 \mathrm{~mA}$, in good agreement with the observed $I_{V P B} \approx 0.5 \mathrm{~mA}$. Having demonstrated the validity of the $I_{V P B}$ concept, this can be used as a tool for obtaining the thickness of the superconducting interface layer in future interfacial superconducting systems.

Referring back to Fig. 1(b), upon further increase of the current beyond the plateau $(I>2 \mathrm{~mA})$, there is once again a non-linear response as the current attains levels that are not only enough to dissociate the vortex-antivortex pairs but are high enough to break Cooper pairs. This is the regular (Cooper) pairbreaking (depairing) regime; here the depairing current $I_{d}(\sim 0.1 \mathrm{~A})$ signals entry into the normal state, upon which $R(I)$ saturates to a constant (residual) value. A detailed investigation of the upper depairing regime was conducted in our earlier work [19].

Fig. 1(a) and (b) show two extreme temperatures, one above $T_{B K T}$ (with $R \rightarrow$ const as $I \rightarrow 0)$ and the other at the bottom temperature of the cryostat and well below $T_{B K T}$ (with $R \rightarrow 0$ as $I \rightarrow 0$ ). To better demarcate the progression that differentiates these behaviors, we measured a complete set of dc $R(I)$ curves at a sequence of temperatures shown in Fig. 2. One observes two qualitative regimes: below some temperature $T_{v} \sim 6 \mathrm{~K}$, the curves are more closely bunched together and show a "fishtail" pattern with a convergence above $I_{V P B} \approx 0.5$ $\mathrm{mA}$; whereas above $T_{v} \sim 6 \mathrm{~K}$, the curves are more widely separated and with decreasing $T$ seem to stall around $\sim 6 \mathrm{~K}$ before falling more rapidly. To better identify this cross over temperature $T_{v}$, a complete temperature-resistance curve was measured at a low value constant current $(53 \mu \mathrm{A})$ corresponding to the left side of the graph in Fig. 2. The result is shown in Fig. 3, which clearly shows a break below at $T_{v}=5.7 \mathrm{~K}$, similar to what was previously observed in MoGe [10]. To qualitatively understand this observation, we first note that the conditions of temperature $\left(T \ll T_{B K T}\right)$ and current $\left(I \ll I_{V P B}\right)$ are such 


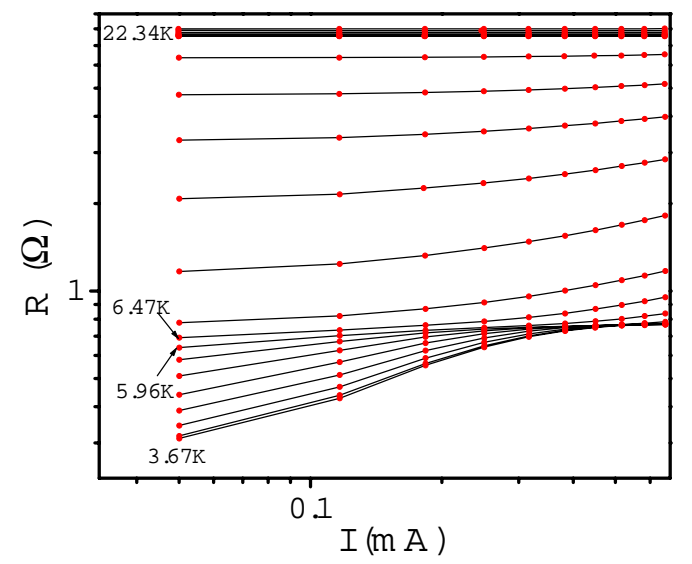

Figure 2: Resistance versus current (dc) curves at various temperatures. The curves below $T \approx 6 \mathrm{~K}$ show a "fishtail" pattern with a convergence above $I_{V P B} \approx 0.5 \mathrm{~mA}$. The temperatures of the curves (lowest to highest) are: 3.67, 3.72, 3.92, 4.22, 4.59, 5.01, 5.46, 5.96, 6.47, 6.98, $7.76,8.56,9.31,10.10,11.00,12.99,14.09,15.26,16.57,18.00,19.34,20.78$, and $22.34 \mathrm{~K}$.

that the BKT mechanism will not provide appreciable dissipation (negligible population of unbound vortex-antivortex pairs). In this case the small amount of residual resistance will arise mainly through the GV process (unbinding of edge nucleated vortices from their antivortex images outside the edge). The GV mechanism is facilitated by the Likharev exploded vortex condition [10], and thus the resistance is expected to decrease more rapidly below the vortexexplosion transition temperature $T_{v}$.

To test the applicability of this Likharev vortex explosion phenomenon to the features observed here at $T_{v}=5.7 \mathrm{~K}$, we use the upper-critical-field measurements of He et al. [12] to estimate the coherence length perpendicular to the plane: we have a perpendicular-to-interface $B_{c 2}^{\perp}(T=5.7 \mathrm{~K})=\Phi_{0} / 2 \pi \xi_{\|}^{2} \approx 7$ $\mathrm{T}$ and a parallel-to-interface $B_{c 2}^{\|}(T=5.7 \mathrm{~K})=\Phi_{0} / 2 \pi \xi_{\|} \xi_{\perp} \approx 27 \mathrm{~T}$, leading to a perpendicular $\xi_{\perp}(T=5.7 \mathrm{~K}) \approx 1.78 \mathrm{~nm}$. Multiplying this by 4.4 gives: $4.4 \xi_{\perp}(T=5.7 \mathrm{~K})=7.8 \mathrm{~nm}$ which agrees well with $7+/-1.1 \mathrm{~nm}$ thickness estimated by He et al. Our measurements thus provide independent evidence for the previously estimated thickness. Or taking that thickness as known (taking it from the $B_{c 2}(T)$ measurement of He et al. as well as from our $I_{V P B}$ measurement of this work), our result becomes a second confirmation of the Likharev vortex explosion phenomenon, having been observed before only once in MoGe films [10].

\section{Conclusions and summary}

We have investigated some novel regimes in the transport behavior of the $\mathrm{Bi}_{2} \mathrm{Te}_{3} / \mathrm{FeTe}$ topological insulator-chalcogenide interface superconductor below 


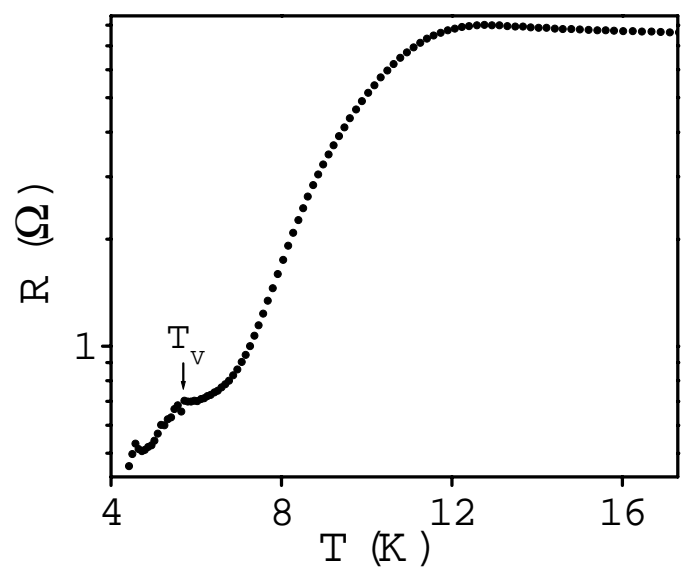

Figure 3: Resistance versus temperature in a low applied current $I=53 \mu \mathrm{A}$. The break occurs at the vortex explosion transition $T_{v}=5.7 \mathrm{~K}$.

the Berezinski-Kosterlitz-Thouless transition. The rather unique sets of experimental curves can be explained through the interplay between current driven unbinding of vortex-antivortex pairs and the Likharev vortex explosion effect. In our earlier work [10] we experimentally demonstrated this phenomenon for the first time and showed its value for estimating superconducting film thickness. In this work we have provided a second demonstration of this effect and used it as a tool to provide an independent confirmation of the superconducting interface layer thickness, which was previously estimated from $B_{c 2}(T)$ measurements. We also demonstrated here the concept of the vortex-pair-breaking current. With the recently growing interest in superconducting interface systems, these effects that occur in such systems are interesting both for their physics novelty as well as their usefulness as investigative tools for improving our understanding of these $2 \mathrm{D}$ systems.

\section{Acknowledgments}

The authors acknowledge useful discussions with A. Gurevich and J. M. Knight. The work related to the measurements and analysis was supported by the U. S. Department of Energy through grant number DE-FG02-99ER45763. The work related to the synthesis of the films was supported by the Research Grants Council of the HKSAR under Grant numbers 16305514 and 16304515.

\section{References}

[1] V. L. Berezinskii, Sov. Phys. JETP 32, 493 (1971).

[2] V. L. Berezinskii, Sov. Phys. JETP 34, 610 (1972). 
[3] J. M. Kosterlitz and D. J. Thouless, J. of Phys. C: Solid State Physics 6, 1181 (1973).

[4] J. M. Kosterlitz, J. Phys. C 7, 1046 (1974).

[5] V. L. Pokrovskii and G. V. Uimin, Sov. Phys. JETP 38, 847 (1974).

[6] J. V. Jose, L. P. Kadanoff, S. Kirkpatrick, and D. R. Nelson, Phys. Rev. B 16, 1217 (1977).

[7] D. R. Nelson and J. M. Kosterlitz, Phys. Rev. Lett. 39, 1201 (1977).

[8] P. B. Wiegmann, J. Phys. C 11, 1583, (1978).

[9] A. Gurevich and V. M. Vinokur, Phys. Rev. Lett. 100, 227007 (2008).

[10] M. N. Kunchur, M. Liang, and A. Gurevich, Phys. Rev. B 86, 024521 (2012).

[11] K. K. Likharev, Rev. Mod. Phys. 51, 101 (1979).

[12] Q. L. He et al. Nature Communications 5, 4247 (2014).

[13] M. N. Kunchur, D. K. Christen, C. E. Klabunde, and J. M. Phillips, Phys. Rev. Lett. 72, 752 (1994).

[14] M. N. Kunchur, Mod. Phys. Lett. B. 9, 399 (1995).

[15] M. N. Kunchur, J. Phys.: Condens. Matter 16, R1183-R1204 (2004).

[16] G. F. Saracila and M. N. Kunchur, Phys. Rev. Lett. 102, 077001 (2009).

[17] G. Blatter et al., Rev. Mod. Phys. 66, 1125 (1994).

[18] A. M. Kadin, K. Epstein, and A. M. Goldman, Phys. Rev. B 27, 6691 (1983).

[19] M. N. Kunchur et al., Phys. Rev. B 92, 094502 (2015). 